\title{
Freezing of parallel hard cubes with rounded edges
}

\begin{abstract}
Matthieu Marechal, ${ }^{1}$ Urs Zimmermann, ${ }^{1}$ and Hartmut Löwen ${ }^{1}$
Institut für Theoretische Physik II: Weiche Materie, Heinrich-Heine-Universität Düsseldorf, Universitätsstraße 1, D-40225 Düsseldorf, Germany
\end{abstract}

(Dated: 25 October 2018)

The freezing transition in a classical three-dimensional system of parallel hard cubes with rounded edges is studied by computer simulation and fundamental-measure density functional theory. By switching the rounding parameter $s$ from zero to one, one can smoothly interpolate between cubes with sharp edges and hard spheres. The equilibrium phase diagram of rounded parallel hard cubes is computed as a function of their volume fraction and the rounding parameter $s$. The second order freezing transition known for oriented cubes at $s=0$ is found to be persistent up to $s=0.65$. The fluid freezes into a simple-cubic crystal which exhibits a large vacancy concentration. Upon a further increase of $s$, the continuous freezing is replaced by a first-order transition into either a sheared simple cubic lattice or a deformed face-centered cubic lattice with two possible unit cells: body-centered orthorhombic or base-centered monoclinic. In principle, a system of parallel cubes could be realized in experiments on colloids using advanced synthesis techniques and a combination of external fields.

\section{INTRODUCTION}

Most liquids freeze into a regular period crystalline lattice upon a sufficient temperature decrease or pressure increase. Since this transition is associated with a breaking of translational symmetry, it is typically discontinuous (or first-order)! ${ }^{1}$ This is in marked contrast to two spatial dimensions where no long-range translational order exists ${ }^{21}$ and the liquid-solid transition can be continuous, for example following the two-stage KosterlitzThouless-Halperin-Nelson-Young scenarid ${ }^{3}$. One of the few (if not the only) exception to the common finding of first-order freezing in three dimensions is a system of parallel hard cubes where a disordered liquid freezes continuously into a simple cubic $(s c)$ lattice at a volume fraction of about 50 percent. This finding was first suggested for parallel hypercubes in more than three spatial dimensions by Kirkpatrick ${ }^{4}$ by using a second order virial expansion. Later, Cuesta and coworkers 58 applied fundamental-measure density functional theory 9910 to parallel hard cubes in three dimensions showing that the freezing transition is also continuous in three dimensions. The continuous nature of the freezing transition was also confirmed later by computer simulations 11112 where the associated criticality was found to be consistent with the Heisenberg universality class ${ }^{12}$

In this paper, we consider a more general shape of hard particles, namely cubes with rounded edges. Our motivation to consider rounded cubes is threefold: First, it is interesting how persistent the second-order transition is with respect to a change of parameters. It is known that orientable cubes (i.e. cubes with full orientational degrees of freedom) freeze via a first-order phase transition $\frac{11113}{1}$ such that additional rotational degrees lead back to the normal picture of freezing. Therefore it is interesting to which extent the degree of rounding affects the order of the transition, in particular, since the extreme hard-sphere limit $d \rightarrow l$ exhibits the common first-order freezing scenario.
Second, fundamental-measure density functional theory (FMT) was developed $\frac{14 / 15}{1}$ and applied $\frac{16 / 17}{17}$ further in recent years towards hard bodies of arbitrary shape. As a version of FMT already exists for the limiting cases, parallel hard cubes and hard spheres, rounded cubes constitute an excellent model system to test the performance of fundamental-measure density functional theory.

Last but not least, it is now possible to fabricate micron-sized colloidal particles with almost arbitrary shapes ${ }^{18}$ 22. In a recent pioneering work of Rossi et al, well-defined colloidal cubes were prepared and studied in real-space by confocal microscopy. These suspensions nicely realize the hard cube model of classical statistical mechanics. In the experimental samples, however, the cubes typically possess rounded edges, therefore our model shape model is closer to these colloids than the hard cube. In the experiments, the colloidal cubes are not oriented in a parallel fashion. Furthermore, nonadsorbing polymers were added to speed up the crystallization process. Therefore, a first-order freezing transition was found in this suspension. Colloidal cubes can in principle be oriented by external aligning field $\$^{2425}$ for instance by introducing an inner core with two distinct non-parallel dipole moments, each of which couples to a separate external field. By simultaneously applying two non-parallel external fields, which could be external electric or magnetic fields or a light field, the orientation of the particle described by its two independent axes can be fixed. We should note here, that the phase behavior of a system of parallel monodisperse particles with only hard-core interactions at constant packing fraction is invariant under scaling of a dimension of the particle by a constant factor. Therefore, it is not inconceivable that our model of parallel rounded cubes would be realized in experiments, possibly as stretched rounded cubes. Furthermore, our work on parallel, rounded, hard cubes provides a good starting point for further studies on colloidal rounded cubes that are not aligned by external fields.

The model for a rounded cube that we use is a sphe- 
rocube, which can be obtained by rounding a cube with edge length $l$. The rounding is done by replacing all edges by quartered cylinders of diameter $d$ and the corners by a spherical octant such that the curvature is continuous on the cube's surface, see Fig. 1. If the diameter $d$ is zero, the traditional model of parallel hard cubes is recovered while for $0<d<l$ we are dealing with truly rounded cubes. Finally, in the extreme limit $d \rightarrow l$ we obtain the hard sphere model where freezing is known to be a firstorder transition into a face-centered-cubic $(f c c)$ lattice. ${ }^{26}$ By splitting the particle surface into planar, cylindrical and spherical parts, we propose a continuous interpolation between a cube and a sphere which is similar in spirit but different in practice to the superball interpolation used recently by Batten et al ${ }^{[27}$ To abbreviate the notation we define a rounding parameter $s=d / l$, similar to Batten et al.'s $1 / q$ for the superballs, in the sense that both $s=1$ and $1 / q=1$ denote a sphere, while $s=0$ and $1 / q=0$ denotes a cube. The overlaps between two superballs can only be detected using an involved numerical algorithm, $\stackrel{27}{2}$ which leads to numerical difficulties as the superball's shape approaches that of a cube. ${ }^{27} \mathrm{In}$ contrast, the overlap algorithm for parallel spherocubes can be given in a closed and very simple form, as we will show in Appendix A. Furthermore, the spherocube is a very convenient model particle for FMT, since the curvatures that feature in the theory are constant on the spherical, cylindrical and planar sections of the particle's surface. Therefore, we have chosen to use the spherocube as our model rounded cube instead of the superball.

We explore the rounded parallel cube model by Monte Carlo (MC) ${ }^{28}$ and event-driven Molecular Dynamics $(\mathrm{EDMD})^{29}$ computer simulations and by fundamental measure density functional theory of freezing ${ }^{9 / 10}$ adjusted conveniently to the rounded shape. As a simulation result, we calculate the equilibrium phase diagram of rounded parallel hard cubes as a function of packing fraction and the degree of rounding embodied in the ratio $s=d / l$. The second order freezing transition known for oriented cubes at $s=0$ is found to be very persistent occurring up to high rounding degrees of about $s=0.65$. This gives evidence that the second-order freezing transition can be seen in experiments on rounded oriented particles. The fluid freezes into a simple-cubic crystal which is accompanied by a very large vacancy concentration in the emerging solid. At further increasing ratios $d / l$, freezing becomes a first-order transition into a sheared $s c$ lattice and a deformed $f c c$ lattice, where the latter can have both orthorhombic (ortho) and monoclinic (clino) unit cells. Our simulation data for the continuous transition line and for the associated vacancy concentration are found to be in qualitative and semi-quantitative agreement with fundamental-measure density functional theory. The three novel crystals (sheared $s c$ and the ortho and clino variants of deformed $f c c$ ) can also be confirmed experimentally and could be useful for designing new materials with novel optical and rheological properties.

The paper is organized as follows: in section III we
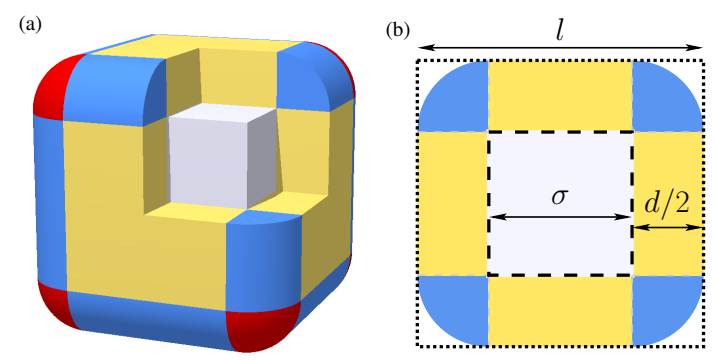

FIG. 1. (a) A spherocube or rounded cube consists of a cube (lightest/gray) surrounded by 6 square prisms (darker/yellow), 12 cylinder sections (still darker/light blue) and 8 spherical sections (darkest/red). Some sections of the outer objects have been removed to show the gray cube. (b) Cross section of the spherocube showing the edge length $\sigma$, minimum radius of curvature $d / 2$, and the total width $l$.

introduce the rounded cube model in detail. We describe the simulation technique in section [III while providing the background of fundamental-measure density functional theory in section IV] Results are presented in section $\mathrm{V}$ and we conclude in section VI

\section{THE MODEL OF ROUNDED PARALLEL HARD CUBES}

Our model rounded cube, the spherocube, is a special case of the sphero-cuboid introduced by Mulder in the context of second order virial theory. ${ }^{[30}$ A spherocube can be obtained by coating a cube with edge length $\sigma$ with a layer of thickness $d / 2$, as shown in Fig. 1. Alternatively, it can be constructed by rounding a larger cube with edge length $l=\sigma+d$ (dotted rectangle in Fig. 1p), such that its edges obtain a nonzero radius of curvature $d / 2$. We will use $s \equiv d / l$ as the shape parameter for the spherocubes. The volume of a rounded cube or spherocube is given by

$$
v_{\mathrm{rc}}=\frac{\pi}{6} d^{3}+\frac{3 \pi}{4} d^{2} \sigma+\sigma^{3}+3 d \sigma^{2} .
$$

In Appendix A, we present the overlap algorithm for parallel spherocubes, which is surprisingly simple, especially compared to the overlap algorithm for superballs.27

The thermodynamic state of the system is sometimes specified using the pressure $P$, but mostly using the volume fraction or packing fraction $\eta \equiv v_{\mathrm{rc}} \rho=v_{\mathrm{rc}} N / V$, where $\rho$ is the density, $N$ the number of particles and $V$ the volume of the system. The temperature $T$ only serves to define the energy unit $k_{B} T$, where $k_{B}$ is Boltzmann's constant.

\section{COMPUTER SIMULATIONS}

In this section, which consists of four parts, the simulations that were performed in this work are described. 
First, we determined candidate crystal structures using a recent, but well-tested simulation technique $\stackrel{31}{,}$ as summarized in the first part of this section. After that we describe the Monte Carlo (MC) and event-driven Molecular dynamics (EDMD) techniques. The structural and thermodynamic properties we measure are listed in the third part and, finally, we describe the methods used to determine the phase behavior in Sec. IIID.

\section{A. Candidate crystal structures}

We find candidate crystal structures by simulating a single unit cell with fully variable box lengths and angles ${ }^{32 / 33}$ in the NPT ensemble, that is the number of particles $N$, the pressure $P$ and the temperature $T$ are held fixed. Using periodic boundary conditions, this unit cell is replicated indefinitely to roughly approximate an infinite crystal. The final configurations of a number of compression series form the unit cells of the potentially stable crystal structures. This computationally inexpensive method has been shown to find all stable crystal phases when applied to a system where the phase behavior was already known ${ }^{31}$ and since then has been employed to find candidate structures for a number of novel systems $34 \sqrt[36]{6}$ and also to find close packed structures. 37

The variant of the method we use is the following: We run a large number of fast compression runs, see Ref. 35 for details. At the lower pressures, the system samples many meta-stable states. As the pressure is quickly increased, the system gets stuck in one of these states. Finally, a nearly perfect crystal is found at very high pressure. To distinguish between different crystals we use the box shape parameter introduced by De Graaf et $a l, \frac{37}{3}$, which is the average length of the box edges times the average area of its faces divided by its volume. The states are divided in clusters, such that the box shape parameter of each state in a certain cluster deviates less than $10^{-4}$ from the box shape parameter of at least one other state in the cluster. The state which used to represent the cluster is the state with the highest density, as this will often be the most ordered one. In the remainder of this work we will refer to this method for determining candidate crystal structures as "unit cell simulations".

The small system size allows large fluctuations in parameters such as the density, which ensure that all possible states are visited. However, the small system size would lead to huge finite size effects, if the results from these simulations would be directly used to determine the region of stability and other thermodynamic properties of the crystals that were found. Rather, this method is intended to be used in concert with conventional simulations (see below), which take crystals formed by replicating the unit cells obtained from this method as initial configurations.

\section{B. Simulation techniques}

We implemented EDMD simulations for hard rounded cubes, which allows us to measure the pressure very efficiently and to quickly equilibrate the system. Molecular dynamics for hard particles is implemented by solving the equation of motion exactly. As such, hard particles perform free motion interrupted by instantaneous collisions in EDMD. Event driven MD simulations are especially fast when collisions can be predicted analytically, such as for hard spheres, and also for the rounded cubes studied in this work, as described in Appendix A.

Although event driven MD simulations are very fast, we found it to be more convenient to use Monte Carlo (MC) simulations in the following situations: Many noncubic crystals show a deformation of the unit cell upon a change in density or pressure. Moves that change the shape of the box ${ }^{32}$ can be easily added to Monte Carlo simulations 33 to account for these deformations. Furthermore, external potentials, such as the ones required for the free energy calculations described further on, can easily be accounted for in Monte Carlo simulations, while they would make the free motion in between collisions too complicated to predict the collisions analytically in EDMD simulations. Finally, we want to allow the vacancy concentration to adjust to changes in the density or pressure. The simplest way to allow the vacancy concentration to change is to allow the box to change its shape in a Monte Carlo simulation. A simulation box which starts with $M_{0} \equiv N_{x}^{(0)} \times N_{y}^{(0)} \times N_{z}^{(0)}$ unit cells with one particle in each unit cell can transform into $M \equiv N_{x} \times N_{y} \times N_{z}$ cells for any integers $N_{x}, N_{y}$ and $N_{z}$, such that $M>M_{0}$. The resulting vacancy concentration is $\nu_{\text {vac }} \equiv 1-M_{0} / M$. In practice, we use a $N_{z}^{(0)}$ which is 50 or 100 , such that the vacancy concentration is at lowest $1-100 / 101 \simeq 0.01$ or $1-50 / 51 \simeq 0.02$. Note, that the minimal vacancy concentration is an order of magnitude larger than the vacancy concentrations in common crystals (for instance the vacancy concentration is of order $10^{-4}$ for hard spheres $\left.\sqrt{38 / 39}\right)$. However, the vacancy concentrations in simple cubic crystals of rounded cubes are orders of magnitude larger than those of hard spheres, as we will see below, which allows us to use this simple technique to measure $\nu_{\mathrm{vac}}$. However, to keep the run time of the simulation limited we have to use considerably smaller system sizes in the other directions: $N_{x}, N_{y}$ are either 10 or 15 . In the other Monte Carlo simulations, we have used a system of approximately 1000 particles unless mentioned otherwise.

\section{Thermodynamic and structural properties}

The order parameter $m$ which measures the degree of crystallinity was introduced by Groh and Mulder 12 It is defined using the maximum of the Fourier transformed density profile for $m_{\nu}$, where $\nu=x, y, z$ denotes a di- 
rection along one of the Cartesian axis and where the density profile is averaged over the two other directions before performing the Fourier transform:

$$
m_{\nu}=\max _{k} \hat{\rho}_{\nu}(k)
$$

The order parameter $m$ is defined by $m=\left(\left|m_{x}\right|+\left|m_{y}\right|+\right.$ $\left.\left|m_{z}\right|\right) / 3$. Because the vacancy concentration and therefore the number of unit cells in each direction can change in the variable box length NPT MC simulations (see Sec. IIIB, we do not know the reciprocal lattice vector $k_{\nu} \hat{x}_{\nu}$, in the $\nu$-direction $\hat{x}_{\nu}$ before hand (However, we do know its direction, because the particles do not rotate). For this reason, we maximize with respect to $k$ in Eq. 2. We can use the resulting length of the wave vector $k_{\nu}$ to determine the number of unit cells in the $\nu$ direction: $N_{\nu}=k_{\nu} L_{\nu} / 2 \pi$, where $L_{\nu}$ is the length of the simulation box in the $\nu$ direction. Obtaining $N_{\nu}$ in all three directions in this way, we can calculate the vacancy concentration $\nu_{\mathrm{vac}}$ as $\nu_{\mathrm{vac}}=1-N /\left(N_{x} N_{y} N_{z}\right)$.

We measure the equation of state using one of two methods depending on the phase of interest. The pressure of the fluid phase as a function of density is measured in NVT EDMD simulations, while the density as a function of the pressure of each of the crystal phases is measured using NPT MC simulations.

We also calculated the mean squared deviation from the lattice position (MSD) to compare to the FMT data. For system with vacancies, the obvious definition of this quantity gives infinity because the particles can easily diffuse away from their lattice position by hopping to a neighboring, empty lattice site. Instead, we measure the MSD from the nearest lattice site. However, we need to know the positions of the perfect lattice sites; specifically, the shift $r_{\nu, 0}$ of the lattice compared to the lattice which has one of its lattice sites in the origin. We use EDMD simulations with zero total momentum. This means that, when a particle hops a lattice constant $a_{0}$ to, say, the left compared to the lattice, the lattice shifts $a_{0} / N$ to the right because the center of mass is fixed. As such the shift $\mathbf{r}_{0}$ drifts with time, and needs to be obtained in the simulation before the MSD can be measured. A reliable way of determining the shift $\mathbf{r}_{0}$ is to use the phase of $\hat{m}_{\nu}\left(k_{\nu}\right)$, which should be equal to $\exp \left(\mathrm{i} k_{\nu} r_{\nu, 0}\right)$.

\section{Phase behavior}

We know from earlier work for perfect cubes 12 and spheres ${ }^{40}$ that the phase diagram contains both first and second order melting transitions. For the first order transitions we use the highly accurate free energy methods that were developed by Frenkel and co-workers. $\frac{41}{\text { We }}$ summarize these methods in Sec. III D 1. The second order phase transitions were located using finite size scaling ${ }^{[12[42]}$ as described in the section after that.

\section{Free energy calculations}

The Helmholtz free energy of the fluid is obtained by integrating the equation of state from the ideal gas limit:

$$
f_{\text {fluid }}^{*}(\rho)=\log \left(\rho l^{3}\right)+\int_{0}^{\rho} \mathrm{d} \rho(P / \rho-1) / \rho
$$

where, here and in Appendix $\mathrm{B}$ the free energy is made dimensionless by $f^{*} \equiv F /\left(N k_{B} T\right)-\log \left(\Lambda^{3} / l^{3}\right), \Lambda$ is the (irrelevant) thermal wavelength $\Lambda=h / \sqrt{2 \pi m_{\mathrm{rc}} k_{B} T}, m_{\mathrm{rc}}$ is the mass of a particle and $h$ is Planck's constant.

The free energies of the crystal phases are measured using the Frenkel-Ladd method ${ }^{28 \mid 41}$ in which the free energy difference between a crystal and the non-interacting Einstein crystal is calculated by thermodynamic integration. We have made some modifications to the method when applying it to the simple cubic crystal phase to allow for a nonzero vacancy concentration. As these modifications are similar to the one applied for rotating cubes in Ref. 13, we leave the details for Appendix B. As an example, the free energy, resulting from the thermodynamic integration technique, is shown as a function of the vacancy concentration in Fig. 2. Clearly, a finite (and quite large) vacancy concentration is found, around $8 \%$. This is surprising, because the packing fraction for this free energy, $\eta=0.53$, is rather high compared to the critical density $\eta_{c} \simeq 0.47$, as determined using the methods described below. We calculated two more free energy curves as a function of vacancy concentration and the resulting vacancy concentrations are shown together which the results from the variable box length $N V T$ simulations and the FMT in Sec.V] Minimizing the free energy with respect to vacancy concentration at every density is somewhat cumbersome, so we have used the variable box length simulations to determine the vacancy fraction in most of this work as described in Sec. IIIC. Once a reference free energy $f^{*}\left(\rho_{0}\right)$ is known at a certain reference density $\rho_{0}$ for each crystal, we integrate over the equation of state $P / \rho^{2}$ to obtain the free energy at all densities, similar to Eq. (3).

When the free energies of all relevant phases at a certain aspect ratio $s$ are known, the coexistence densities for a given pair of phases 1 and 2 can be found by solving $P_{1}\left(\rho_{1}\right)=P_{2}\left(\rho_{2}\right)$ and $\mu_{1}\left(\rho_{1}\right)=\mu_{2}\left(\rho_{2}\right)$, where the pressure $P_{i}$ of phase $i$ is obtained from a fit of the equation of state and the chemical potential $\mu_{i}=F_{i} / N_{i}+P_{i} / \rho_{i}$. Solving these equations for all possible pairs of phases and finding at each density the phase or phase coexistence, which has the lowest free energy, the phase diagram can be drawn.

\section{Finite size scaling}

For a second order phase transition, the above method can not be used due to very large finite size effects near the transition. Near the phase transition, we can use finite size scaling $\sqrt[42]{ }$ to find the properties of the infi- 


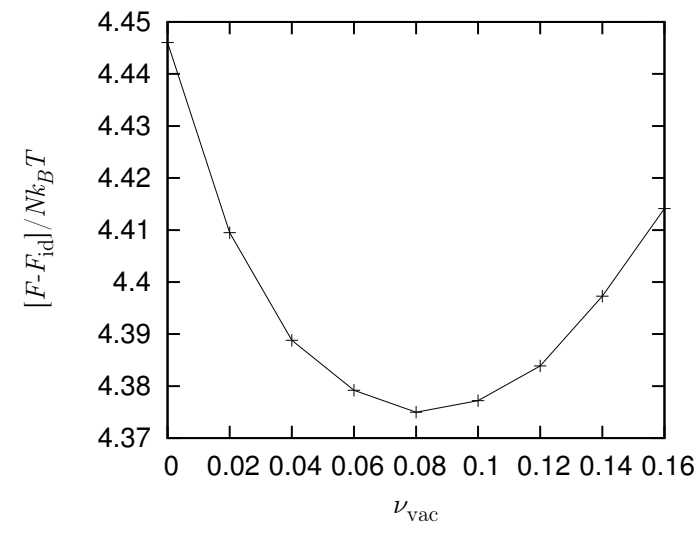

FIG. 2. The free energy of a simple cubic crystal of spherocubes with $s=0.6$ as a function of its vacancy fraction $\nu_{\mathrm{vac}}$ at $\eta=0.53$ for $N \simeq 1000$ particles.

nite system. Only the behavior of the order parameter with pressure and system size is required to find the location of the transition; no thermodynamic integration is required in this case. We use the scaling of $\langle m\rangle=N^{-\nu_{1}} \tilde{m}\left(\left|P_{c} / P-1\right| N^{\nu_{2}}\right)$ and the Binder cumulant ${ }^{42} U_{N} \equiv 1-\left\langle m^{4}\right\rangle /\left\langle m^{2}\right\rangle^{2}=\tilde{U}\left(\left|P_{c} / P-1\right| N^{\nu_{2}}\right)$, where $P_{c}$ is the critical pressure, $\nu_{1}$ and $\nu_{2}$ are finite size scaling exponents and $\tilde{m}$ and $\tilde{U}$ are scaling functions. The exponents $\nu_{1}$ and $\nu_{2}$ fall into certain universality classes (In terms of the critical exponents $\beta$ and $\nu$ often used in the literature, $\nu_{1}=-\beta / 3 \nu$ and $\left.\nu_{2}=1 / 3 \nu\right)$. Groh and Mulder ${ }^{12}$ determined the universality class for the melting transition of hard cubes without vacancies to be that of the three dimensional classical Heisenberg model, which has $\nu_{1} \simeq 0.173$ and $\nu_{2} \simeq 0.472 \cdot{ }^{43}$ We have not found any evidence that these exponents are changed when vacancies are included and, therefore, use these values for the exponents also here.

We used the system sizes $N=10^{3}, 15^{3}$ and $20^{3}$, which are large enough that we can neglect corrections to finite size scaling ${ }^{\sqrt{12}}$ (our large system size is considerably larger than that of Ref. 12).

A method to determine the critical pressure that does not use the values of the scaling exponents consists of plotting $U_{N}$, which does not depend on system size for $P=P_{c}$, for a number of system sizes. The point where the three curves meet is the critical pressure $P_{c}$. We used this method for $s=0$ and $s=0.5$. When $N^{\nu_{1}}\langle m\rangle$ is plotted against $\left|P_{c} / P-1\right| N^{\nu_{2}}$ the curves fall on top of a single master curve, which confirms the finite size scaling ansatz that $\tilde{m}\left(\left|P_{c} / P-1\right| N^{\nu_{2}}\right) \equiv\langle m\rangle N^{\nu_{1}}$ is a universal function of the relative deviation from the critical pressure. We exploited the universalness of this function to determine $P_{c}$ for the values $s=0.1,0.2,0.3,0.4,0.6$ and 0.65 by fitting $P_{c}$ such that the data for $\tilde{m}\left(\left|P_{c} / P-1\right| N^{\nu_{2}}\right)$ fall on top of a single master curve for all values of $s$ and system sizes considered. The collapse is shown in Fig. 3 . Note, that the vacancy concentration changes as a function of

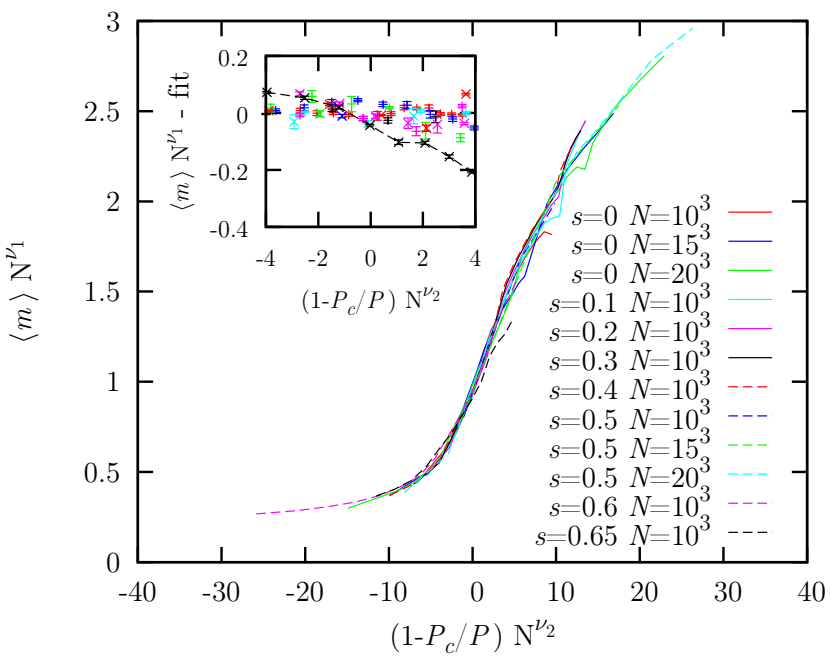

FIG. 3. The scaled positional order parameter, $\langle m\rangle N^{\nu_{1}}$, of systems of $N$ hard rounded parallel cubes with $s=0,0.10 .2$ 0.30 .40 .50 .6 and 0.65 as a function of $\left(1-P / P_{c}\right) N^{\nu_{2}}$, where $P_{c}$ is the critical pressure (that is a function of $s$ ) and $\nu_{1}$ and $\nu_{2}$ are scaling exponents. The system size is $N=10^{3}$ for the results for $s=0$ and $s=0.5$, for which $N=10^{3}, 15^{3}$ and $20^{3}$. The inset shows $\langle m\rangle N^{\nu_{1}}$ near the critical pressure with a fit to the results for $s=0$ and $N=10^{3}$ subtracted for clarity. The pluses and crosses correspond to the solid and dashed lines, respectively, of the same color in the main plot.

the pressure. Consequently, the number of unit cells in a certain direction changes discretely in these simulations, which is the cause of the noise in Fig. 3. Reassuringly, the collapse of the positional order parameters is reasonably good, considering the noise. The inset shows a zoom near the critical pressure and a fit to the data for $s=0$ and $N=10^{3}$ has been subtracted for clarity. Most of the data in the inset is indeed scattered around zero for a range of pressures near $P=P_{c}$, where the exception seems to be $s=0.65$ (black dashed line). Apparently, corrections to scaling are more important for this value of $s$, which is close to the triple point where the simple cubic phase is replaced by another crystal phase. Therefore, the critical pressure $P_{c}$ for $s=0.65$ is less accurate than $P_{c}$ for the other values for $s$.

\section{FUNDAMENTAL-MEASURE DENSITY FUNCTIONAL THEORY}

In the framework of density functional theory 44 the equilibrium grand canonical potential is obtained by minimizing the density functional

$$
\Omega[\rho]=\mathcal{F}[\rho]+\int \mathrm{d} \mathbf{r} \rho(\mathbf{r})\left(V_{\mathrm{ext}}(\mathbf{r})-\mu\right),
$$

with the intrinsic free energy functional $\mathcal{F}[\rho]$, the external potential $V_{\text {ext }}(\mathbf{r})$, the density distribution $\rho(\mathbf{r})$ and 
the chemical potential $\mu$. We limit our considerations to a one-component system but the theory can easily be generalized to multicomponent systems. The functional $\mathcal{F}[\rho]$ naturally separates into two parts, $\mathcal{F}[\rho]=\mathcal{F}_{\text {id }}[\rho]+\mathcal{F}_{\text {exc }}[\rho]$ with the ideal gas contribution

$$
\mathcal{F}_{\mathrm{id}}[\rho]=k_{\mathrm{B}} T \int \mathrm{d} \mathbf{r} \rho(\mathbf{r})\left(\log \left(\rho(\mathbf{r}) \Lambda^{3}\right)-1\right),
$$

where $\Lambda$ is the thermal wavelength as defined above. The excess free energy $\mathcal{F}_{\text {exc }}[\rho]$ which contains the information of particle interactions is not exactly known, such that one has to rely on approximations.

For hard sphere systems, Rosenfeld's fundamental measure theory (FMT) $)^{9}$ and refined versions of FMT $45 \mid 46$ are currently the most accurate density functional approaches 47 . Rosenfeld $\frac{48}{4}$ also generalized the FMT to arbitrarily convex shaped hard interacting particles using the Gauss-Bonnet theorem. His theory yields good results only for mildly elongated particles 49 . Recently, Hansen-Goos and Mecke improved these considerations ${ }^{14 \mid 15}$ within the so-called extended deconvolution fundamental measure theory (edFMT). First, we briefly review the results of edFMT and subsequently apply it to spherocubes.

\section{A. General approach of edFMT}

In the low density limit, we can express the excess free energy functional as a second order virial expansion:

$$
\lim _{\rho \rightarrow 0} \mathcal{F}_{\text {exc }}[\rho]=-\frac{k_{\mathrm{B}} T}{2} \iint \mathrm{d} \mathbf{r}^{\prime} \mathrm{d} \mathbf{r} \rho\left(\mathbf{r}^{\prime}\right) \rho(\mathbf{r}) f\left(\mathbf{r}-\mathbf{r}^{\prime}\right) .
$$

Here $\left.f(\mathbf{r})=\exp \left(-\varphi(\mathbf{r}) / k_{\mathrm{B}} T\right)\right)-1$ is the Mayer function with pair interaction potential $\varphi$ between two particles. In the context of hard-body interactions the Mayer function simply reads $f(\mathbf{r})=-1$ for overlapping particles and $f(\mathbf{r})=0$ otherwise. The Mayer function can be deconvoluted into a sum of weight functions $w_{\alpha}$ that capture the geometrical features of a single convex particle, as shown in 1415 :

$$
\begin{aligned}
-\frac{f(\mathbf{r})}{2}= & w_{0} \otimes w_{3}(\mathbf{r})+w_{1} \otimes w_{2}(\mathbf{r})-\mathbf{w}_{1} \otimes \mathbf{w}_{2}(\mathbf{r}) \\
& -\sum_{j=2}^{\infty}(-1)^{j} W_{1}^{[j]} \otimes W_{2}^{[j]}(\mathbf{r}) .
\end{aligned}
$$

Here and in the remainder, we will denote scalar quantities by $x$, vector quantities by $\mathbf{x}$ and tensors of rank $j \geq 2$ by $X^{[j]}$. The entire set of related scalar, vectorial and tensorial quantities is referred to as $\left\{x_{\alpha}\right\}$. The operation $\otimes$ of two weight functions is defined by

$$
w_{\alpha} \otimes w_{\gamma}(\mathbf{r})=\int \mathrm{d} \mathbf{r}^{\prime} w_{\alpha}\left(\mathbf{r}^{\prime}\right) \star w_{\gamma}\left(\mathbf{r}^{\prime}-\mathbf{r}\right) .
$$

Here, we use the generalized scalar product $\star$, which is meant to be a multiplication for scalar quantities, scalar product for vector quantities and trace of the product of two matrices for tensors of second order. In general, for tensors of $j$ th order we have:

$$
X^{[j]} \star Y^{[j]}=\sum_{i_{1}, \ldots, i_{j}}\left(X^{[j]}\right)_{i_{1} \ldots i_{j}} \cdot\left(Y^{[j]}\right)_{i_{j} \ldots i_{1}} .
$$

The geometrical weight functions $\left\{w_{\alpha}\right\}$ are given by

$$
\begin{aligned}
w_{3}(\mathbf{r}) & =\Theta(|\mathbf{R}(\hat{\mathbf{r}})|-|\mathbf{r}|) \\
w_{2}(\mathbf{r}) & =\frac{\delta(|\mathbf{R}(\hat{\mathbf{r}})|-|\mathbf{r}|)}{\hat{\mathbf{n}} \cdot \hat{\mathbf{r}}} \\
w_{1}(\mathbf{r}) & =\frac{H(\mathbf{r})}{4 \pi} w_{2}(\mathbf{r}) \\
w_{0}(\mathbf{r}) & =\frac{K(\mathbf{r})}{4 \pi} w_{2}(\mathbf{r}) \\
\mathbf{w}_{2}(\mathbf{r}) & =\hat{\mathbf{n}} w_{2}(\mathbf{r}) \\
\mathbf{w}_{1}(\mathbf{r}) & =\frac{H(\mathbf{r})}{4 \pi} \mathbf{w}_{2}(\mathbf{r}) \\
W_{1}^{[2]}(\mathbf{r}) & =\frac{\Delta \kappa(\mathbf{r})}{4 \pi}\left(\mathbf{v}^{\mathrm{I}} \mathbf{v}^{\mathrm{I}}(\mathbf{r})-\mathbf{v}^{\mathrm{II}} \mathbf{v}^{\mathrm{II}}(\mathbf{r})\right) w_{2}(\mathbf{r}) \\
W_{2}^{[2]}(\mathbf{r}) & =\hat{\mathbf{n}}(\mathbf{r}) \hat{\mathbf{n}}^{T}(\mathbf{r}) w_{2}(\mathbf{r}) \\
\left(W_{1}^{[j]}\right)_{i_{1} \ldots i_{j}} & \frac{\Delta \kappa(\mathbf{r})}{4 \pi}\left(v_{i_{1}}^{\mathrm{I}} v_{i_{2}}^{\mathrm{I}}-v_{i_{1}}^{\mathrm{II}} v_{i_{2}}^{\mathrm{II}}\right) \hat{n}_{i_{3}} \cdots \hat{n}_{i_{j}} w_{2}(\mathbf{r}) \\
\left(W_{2}^{[j]}\right)_{i_{1} \ldots i_{j}} & =\hat{n}_{i_{1}} \hat{n}_{i_{2}} \cdots \hat{n}_{i_{j}} w_{2}(\mathbf{r}) .
\end{aligned}
$$

In this notation, $\mathbf{R}(\hat{\mathbf{r}})$ is the vector that points along the direction $\hat{\mathbf{r}}$ from a certain reference point inside a particle to its surface. The principle curvatures $\kappa_{\mathrm{I}}$ and $\kappa_{\text {II }}$ with corresponding principle directions $\mathbf{v}^{\mathrm{I}}$ and $\mathbf{v}^{\mathrm{II}}$ are defined at $\mathbf{R}(\hat{\mathbf{r}})$. We also define the mean curvature $H=\left(\kappa_{\mathrm{I}}+\kappa_{\mathrm{II}}\right) / 2$, the Gaussian curvature $K=\kappa_{\mathrm{I}} \kappa_{\mathrm{II}}$ and the deviatoric curvature $\Delta \kappa=\left(\kappa_{\mathrm{I}}-\kappa_{\mathrm{II}}\right) / 2$ for convenience. The normal vector is written as $\hat{\mathbf{n}}$. With $\mathbf{a}^{T}$ we denote the transpose of the vector or matrix. Consequently, $\mathbf{a} \mathbf{b}^{T}$ is the dyadic product of vectors $\mathbf{a}$ and b.

Finally, we introduce weighted densities as a convolution of the density profile with the corresponding weight function:

$$
n_{\alpha}(\mathbf{r})=\int \mathrm{d} \mathbf{r}^{\prime} \rho\left(\mathbf{r}^{\prime}\right) w_{\alpha}\left(\mathbf{r}-\mathbf{r}^{\prime}\right) .
$$

In terms of weighted densities the low density limit 4 becomes

$$
\begin{gathered}
\lim _{\rho \rightarrow 0} \mathcal{F}_{\text {exc }}[\rho]=k_{\mathrm{B}} T \int \mathrm{d} \mathbf{r}\left[n_{0} n_{3}+n_{1} n_{2}-\mathbf{n}_{1} \cdot \mathbf{n}_{2}\right. \\
\left.-\sum_{j=2}^{J} \zeta_{j} N_{1}^{[j]} \star N_{2}^{[j]}\right]
\end{gathered}
$$

The tensorial weighted density $N_{\alpha}^{[j]}$ should not be confused with the number of particles $N$. Here, we truncated the tensor expansion after the $J$ th term and introduced 
the free parameters $\zeta_{j}$. In the limit $J \rightarrow \infty$, the exact low density limit is recovered provided $\zeta_{j}=(-1)^{j}$. The tensorial terms account for the asphericity and vanish only for hard sphere particles. In their original work on edFMT, ${ }^{14}$ Hansen-Goos and Mecke truncated the infinite sum at $J=2$. Subsequently, they showed, for an anisotropic fluid of spherocylinders, that renormalization by $\zeta_{2} \neq 1$ better corrects for the influence of the truncation than including higher order tensorial terms without renormalizing (i.e. with $\zeta_{2}=1$ ) ${ }^{15}$

In edFMT the following ansatz is made for the excess free energy

$$
\mathcal{F}_{\text {exc }}[\rho]=k_{\mathrm{B}} T \int \mathrm{d} \mathbf{r} \Phi\left[\left\{n_{\alpha}(\mathbf{r})\right\}\right],
$$

where the free energy density $\Phi$ solely depends on the set of weighted densities $\left\{n_{\alpha}\right\}$. Clearly, as $\rho \rightarrow 0$ we should recover (7). As a result of scaled particle theory and dimensional analysis, the free energy density for truncated tensor terms reads

$$
\Phi=-n_{0} \log \left(1-n_{3}\right)+\frac{\phi_{1}\left(\left\{n_{\alpha}\right\}\right)}{1-n_{3}}+\frac{\phi_{2}\left(\left\{n_{2}\right\}\right)}{\left(1-n_{3}\right)^{2}},
$$

with

$$
\begin{aligned}
\phi_{1}\left(\left\{n_{\alpha}\right\}\right)= & n_{1} n_{2}-\mathbf{n}_{1} \cdot \mathbf{n}_{2}-\sum_{j=2}^{J} \zeta_{j} N_{1}^{[j]} \star N_{2}^{[j]} \\
\phi_{2}\left(\left\{n_{2}\right\}\right)= & c_{0}\left(\mathbf{n}_{2}^{T} N_{2}^{[2]} \mathbf{n}_{2}-n_{2} \mathbf{n}_{2} \cdot \mathbf{n}_{2}\right. \\
& \left.+n_{2} \operatorname{Tr}\left[\left(N_{2}^{[2]}\right)^{2}\right]-\operatorname{Tr}\left[\left(N_{2}^{[2]}\right)^{3}\right]\right) .
\end{aligned}
$$

The trace of a matrix $X^{[2]}$ is denoted as $\operatorname{Tr}\left[X^{[2]}\right]$. The $\phi_{2}$ term was introduced by Tarazon ${ }^{50}$ within a dimensional crossover analysis ${ }^{10}$ In the edFMT $c_{0}=3 /(16 \pi)$ was chosen. In this way the exact third virial coefficient of hard spheres is included. For general convex rotating (i.e. non-parallel) particles, the edFMT is exact up to the second virial coefficient for the isotropic fluid.

\section{B. edFMT of parallel hard spherocubes}

We now apply the edFMT to a monocomponent system of parallel hard spherocubes. We chose the model of spherocubes as the curvatures of all sections of its surface are constant, where we divided the surface of the particle into its spherical, cylindrical and flat components, see Fig. 1. It is thus sensible to split the weight functions into a sum of contributions, each of which is related to one of these sections of the surface or, for $n_{3}$, to the corresponding volume. Since the convolution is a bilinear operation, the weighted densities also decompose into a sum of terms related to the different components. Due to this decomposition we are able to determine the weighted densities for each component separately and use a coordinate system appropriate for its geometry.

\section{Homogeneous fluid}

As the most simple case, we first study the monocomponent homogeneous fluid. It is characterized by a constant density profile $\rho(\mathbf{r}) \equiv N / V$. Consequently, the weighted densities $\left\{n_{\alpha}\right\}$ are independent of the position vector and read $n_{\alpha}=\rho m_{\alpha}$, with $m_{\alpha}=\int \mathrm{d} \mathbf{r} w_{\alpha}$. The integrated scalar weight functions $m_{\alpha}$ for $\alpha=3,2,1,0$ represent the volume, surface, mean half width 51 and Euler characteristic of a spherocube. Furthermore, we notice that the packing fraction $\eta$ is equal to $n_{3}$. Thus, we can express the weighted densities in dependence of the packing fraction as

$$
n_{\alpha}=\eta \frac{m_{\alpha}}{m_{3}} .
$$

In the case of the scalar weighted densities we obtain

$$
\begin{aligned}
& m_{0}=1 \\
& m_{1}=\frac{1}{4}(3 l-d) \\
& m_{2}=\pi d^{2}+3 \pi d \sigma+6 \sigma^{2} \\
& m_{3}=v_{\mathrm{rc}}
\end{aligned}
$$

The $\mathbf{n}_{2}$ vector-type weighted density vanishes for the homogeneous fluid, which is a consequence of the Gauss' divergence theorem and holds for arbitrarily shaped particles $\frac{15}{15}$ As a result, the vector term in the free energy density (8) vanishes. Additionally, the tensor weighted density $N_{1}^{2]}$ is zero due to the cubic symmetry of the particle and the traceless nature of $N_{1}^{[2]}$. As a result, truncation at $J=2$ order would leave us only with the scalar terms, which does not result in the correct second virial coefficient. To improve the theory, we need to extend (5) at least to the first tensor term that does not vanish in the homogeneous fluid. In the case of spherocubes, the first nonzero term contains tensors of fourth order, i.e. $J=4$. The corresponding generalized product reads

$$
N_{1}^{[4]} \star N_{2}^{[4]}=\frac{\eta^{2}}{m_{3}^{2}}\left(\frac{3}{8} \sigma^{3}+\frac{9}{128} \pi d \sigma^{2}\right) .
$$

In this way we can determine the free parameter $\zeta_{4}$ by comparison with the exact second virial coefficient $B_{2}$. The virial expansion up to the third virial coefficient $B_{3}$ reads

$$
\frac{P}{\rho k_{\mathrm{B}} T}=1+\frac{B_{2}}{v_{\mathrm{rc}}} \eta+\frac{B_{3}}{v_{\mathrm{rc}}^{2}} \eta^{2}+\mathcal{O}\left(\eta^{3}\right),
$$

with $B_{2}=4 v_{\mathrm{rc}}$ for spherocubes as can be shown by elementary geometrical considerations. On the other hand, the compressibility factor is given by the derivation of the free energy density with respect to $n_{3}$ :

$$
\frac{P}{\rho k_{\mathrm{B}} T}=\frac{\eta}{N} \frac{\partial \mathcal{F}}{\partial \eta}=\frac{1+a\left(\zeta_{4}\right) \eta+b\left(\zeta_{4}, c_{0}\right) \eta^{2}}{(1-\eta)^{3}},
$$


where

$$
\begin{aligned}
a\left(\zeta_{4}\right) & =\frac{m_{1} m_{2}-\zeta_{4} M_{1}^{[4]} \star M_{2}^{[4]}}{m_{3}}-2, \\
b\left(\zeta_{4}, c_{0}\right) & =c_{0} \frac{4 m_{2}^{3}}{9 m_{3}^{2}}-a\left(\zeta_{4}\right)-1 .
\end{aligned}
$$

The Taylor expansion of Eq. 12 around $\eta=0$ is

$$
\frac{P}{\rho k_{\mathrm{B}} T}=1+(a+3) \eta+(3 a+b+6) \eta^{2}+\mathcal{O}\left(\eta^{3}\right),
$$

where we dropped the $\zeta_{4}$ and $c_{0}$ dependences for brevity. By comparing the terms of order $\eta$ in (12) and (11) with each other, we obtain $a\left(\zeta_{4}\right)=1$. Solving this equation for $\zeta_{4}$ yields

$$
\zeta_{4}=\frac{m_{1} m_{2}-3 m_{3}}{M_{1}^{[4]} \star M_{2}^{[4]}} .
$$

The free parameter $\zeta_{4}$ only depends on the shape parameter $s$.

In the limit of hard parallel cubes $(s=0)$, we do not recover the equation of state found by Cuesta and Martínez-Ratón, ${ }^{[]}$because for non-spherical shapes the third virial coefficient is not exact. We can resolve this issue by adjusting the constant $c_{0}$, which was introduced in (10). Equating the third virial coefficient in (11) with the one in (13) and inserting $a\left(\zeta_{4}\right)=1$, yields $b\left(\zeta_{4}, c_{0}\right)=B_{3} v_{\mathrm{rc}}^{-2}-9$, which is equivalent to

$$
c_{0}=\frac{9 m_{3}^{2}}{4 m_{2}^{3}}\left(\frac{B_{3}}{v_{\mathrm{rc}}^{2}}-7\right) .
$$

In contrast to $B_{2} / v_{\mathrm{rc}}=4$, the dimensionless third virial coefficient $B_{3} / v_{\mathrm{rc}}^{2}$ depends on the shape parameter and has to be determined for every $s$. We have numerically calculated the third virial coefficient using Monte-Carlo integration with an approximate error of $\approx 10^{-4}$. The result $B_{3}(s)$ smoothly interpolates between the analytically known $B_{3}$ for cubes $B_{3}(0)=9 v_{\mathrm{rc}}^{2[52]}$ and spheres $B_{3}(1)=10 v_{\mathrm{rc}}^{2}{ }^{53}$ Using the respective third virial coefficients of these limiting cases, we recover the FMT equations of state of spheres and parallel hard cubes.

\section{Simple cubic crystal}

We parametrize the density profile of the crystal by a standard Gaussian form given by

$$
\rho\left(\alpha, \nu_{\mathrm{vac}}, \mathbf{r}\right)=\left(1-\nu_{\mathrm{vac}}\right)\left(\frac{\alpha}{\pi}\right)^{\frac{3}{2}} \sum_{\mathbf{R}} \exp \left(-\alpha(\mathbf{r}-\mathbf{R})^{2}\right),
$$

where $\{\mathbf{R}\}$ are the lattice vectors of the prescribed crystal structure. We regard $\nu_{\mathrm{vac}} \in[0,1]$ as the vacancy concentration and $\alpha \in[0, \infty)$ as the Gaussian parameter that characterizes the profile. For a simple cubic crystal structure the parametrization factorizes and takes a simpler form with lattice constant $a_{0}=\left(\left(1-\nu_{\mathrm{vac}}\right) m_{3} / \eta\right)^{\frac{1}{3}}$, provided that $\left(1-\nu_{\text {vac }}\right) m_{3} \geq \eta l^{3}$. With this parametrization we can determine the weighted densities according to (6). As for the homogeneous fluid, we truncate the tensor expansion at $J=4$. For inhomogeneous density distributions, the generalized products of the second and third order tensors in general do not vanish. Thus, we need to determine the free parameters $\zeta_{2}$ and $\zeta_{3}$. In the special case $s=0$, the infinite tensor expansion in Eq. (9) with $J=\infty$ and $\zeta_{j}=(-1)^{j}$ can be evaluated analytically for the simple cubic crystal, which gives the same result as the truncated $\phi_{1}$ in Eq. 9 with $\left(\zeta_{2}, \zeta_{3}, \zeta_{4}\right)=(1,-3,4)$ for $J=4$. In this work, we require that the truncated free energy functional is identical to this analytical free energy functional for the simple cubic crystal in the limit $s \rightarrow 0$. Accordingly, we set $\left(\zeta_{2}, \zeta_{3}, \zeta_{4}\right)=\left(1,-3, \zeta_{4}\right)$ for spherocubes with finite $s$, where $\zeta_{4}$ is given by the value for the homogeneous fluid (14). Numerical minimization of the free energy functional with respect to $\alpha$ and $\nu_{\text {vac }}$ yields a continuous freezing transition for $s \leq 0.65$.

\section{RESULTS}

\section{A. Crystals and regular close packing}

We found two different types of crystals in the unit cell simulations described in Sec. IIIA. The crystal found at low aspect ratios (for near cubes) resembles a sheared version of the simple cubic phase, see Fig. 4(a). The (primitive) lattice vectors at close packing are given by

$$
\mathbf{a}_{1}=\left(\begin{array}{c}
l \\
\Delta a \\
\Delta a
\end{array}\right), \quad \mathbf{a}_{2}=\left(\begin{array}{c}
\Delta a \\
l \\
\Delta a
\end{array}\right), \text { and } \mathbf{a}_{3}=\left(\begin{array}{c}
\Delta a \\
\Delta a \\
l
\end{array}\right)
$$

where $\Delta a$ is given by $\Delta a \equiv d(1-1 / \sqrt{2})$. The Bravais lattice of the sheared simple cubic phase (shSC) is the rhombohedral lattice. At close packing, the packing fraction of the shSC crystal is

$$
\eta_{\mathrm{shSC}}=v_{\mathrm{rc}} /\left\{l^{3}-3 \Delta a^{2} l+2 \Delta a^{3}\right\} .
$$

The shSC crystal has the highest packing for $s<$ 0.781133; for higher aspect ratios, a phase similar to face centered cubic was encountered, which we called deformed FCC (def FCC), also depicted in Fig. 4(a). This phase actually consists of two phases between which a continuous transition is observed. Decreasing $s$ from 1 (that is starting with spheres), FCC is deformed such that only a base centered monoclinic (BCM) unit cell can be recognized (a BCM unit cell can also found in the cubic unit cell of FCC). As the aspect ratio is decreased beyond 0.825079 , a body-centered orthorhombic unit cell is found to have the highest packing fraction. Both crystals can be described using the BCM unit cell (which is 
(a)

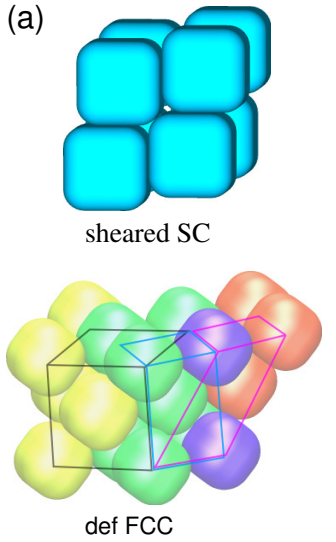

(b)
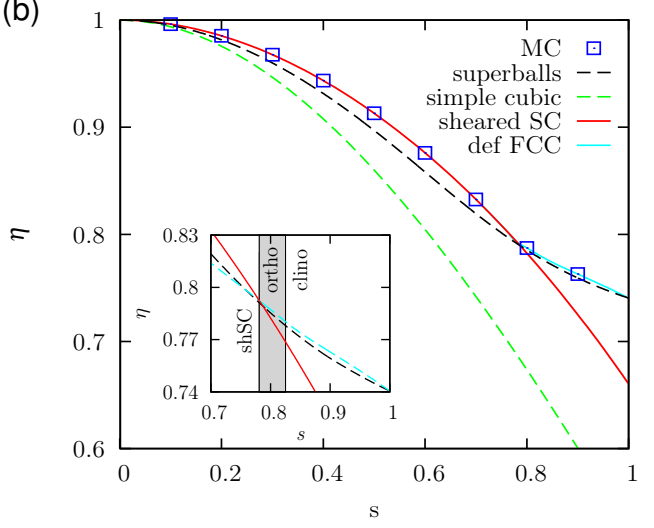

(c)

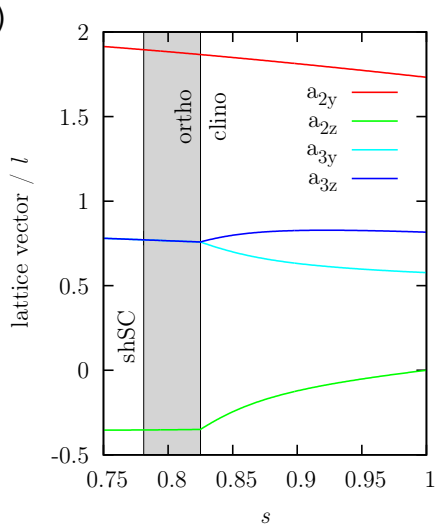

FIG. 4. (a) The crystals that were found using the unit cell simulations: a "sheared" version of a simple cubic crystal (shSC) and a deformed face-centered cubic crystal (def FCC). For the deformed FCC crystal, the deformed cubic unit cell of FCC is indicated in black (yellow and green particles), the body centered orthorhombic (ortho) unit cell in blue (green and blue particles) and the base centered monoclinic (clino) unit cell in magenta (red, blue and some of the green particles). (b) The packing fractions of the various crystals as a function of the aspect ratio $s$ at close packing. For comparison, the packing fraction at close packing for superballs which are mapped onto spherocube with aspect ratio $s$ (see Appendix C) are included, as well as the packing fraction of the simple cubic phase. The inset shows an enlargement of the region where the def FCC phase has the highest packing. (c) The lattice vectors of the ortho and clino variants of def FCC. In (b) and the inset of (c) the region where the various phases have the highest packing are indicated by the labels "shSC", "ortho" and "clino" and the gray area.

the most general):

$$
\mathbf{a}_{1}=\left(\begin{array}{c}
l \\
0 \\
0
\end{array}\right), \quad \mathbf{a}_{2}=\left(\begin{array}{c}
0 \\
a_{2 y} \\
a_{2 z}
\end{array}\right), \text { and } \mathbf{a}_{3}=\left(\begin{array}{c}
0 \\
a_{3 y} \\
a_{3 z}
\end{array}\right),
$$

where $a_{i \nu}$ for $i=2,3$ and $\nu=y, z$ are to be determined and the 'base' of the unit cell, on which both particles in the unit cell lie, is spanned by $\mathbf{a}_{1}$ and $\mathbf{a}_{2}$. The components $a_{i \nu}$ in general have to be calculated numerically. The ones at close packing are plotted in Fig. 4(c).

The packing fraction at close packing for these crystals are shown in Fig. 4(b). For comparison, the close-packed simple cubic crystal is also shown. Clearly, the simple cubic phase has a lower maximal packing fraction than the sheared simple cubic phase for $s>0$ and, naively, one would think that the sheared simple cubic crystal is more stable for all densities. However, we will show below that the fluid first transforms into a simple cubic crystal phase as the pressure is increased for a large range of $s$ values. This shows once again that packing arguments should not be used to infer the stable crystal at finite pressures. We also included the maximal packing fraction for superballs in Fig. 4(b) as a function of the $s$-value of the spherocube that has a minimal Hausdorff distance to the superball, see Appendix C. Cube-like superballs show two distinct crystal phases at close packing, 54 which are quite similar to our shSC and def FCC phases. Clearly, superballs have a lower packing fraction at close packing for all values of $s$, which is especially marked at low $s$, where the flat faces of the spherocubes allow a very efficient packing into the shSC phase.

\section{B. Comparison between FMT and simulations}

In this section we compare the data from the Monte Carlo and event-driven MD simulations to FMT results for the simple cubic crystal. In Figs. 5(a) and (b), we show the vacancy concentration as measured in variable box length $N V T$ Monte Carlo simulations and compare with the results from FMT. Also shown are two black points, which are determined from free energy calculations. Reassuringly, these points correspond well to the other simulation results. Fig. 5(a) shows the dependence on the packing fraction for fixed aspect ratio $s=0$. The trend of the vacancy concentration from edFMT corresponds to that of the $\mathrm{MC}$ simulations, as does the original FMT for hard parallel cubes by Cuesta et $a i^{\sqrt{5}}$. However, both theories underestimate the vacancy concentration considerably. Possibly, fluctuations, which are absent in the theory, stabilize crystals with higher vacancy concentrations. The dependence of the vacancy concentration on the aspect ratio $s$ is shown in Fig. 5(b). The functional by Cuesta et $a \sqrt{5}$ cannot be applied for rounded hard cubes with $s \neq 0$. While the simulation data shows only a very weak dependence on $s$, the theoretical result increases dramatically with increasing $s$, such it actually overestimates the data at $s \gtrsim 0.1$. Nevertheless, both theory and simulations show that the high vacancy concentration is not an artifact of the sharp edges of the parallel hard cubes, but remains also when the edges are rounded.

In Fig. 6, the equation of state $\left(P / \rho k_{B} T\right)$ in the homogeneous fluid and in the simple cubic crystal with the vacancy concentration of Fig. 5 is shown. Again, simula- 
(a)

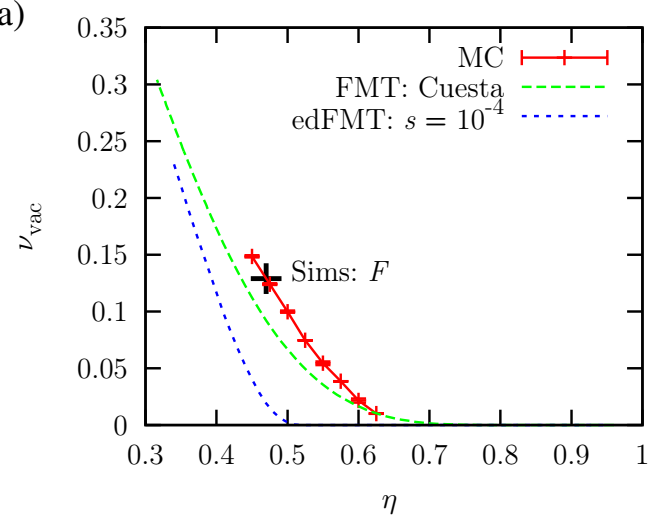

(b)

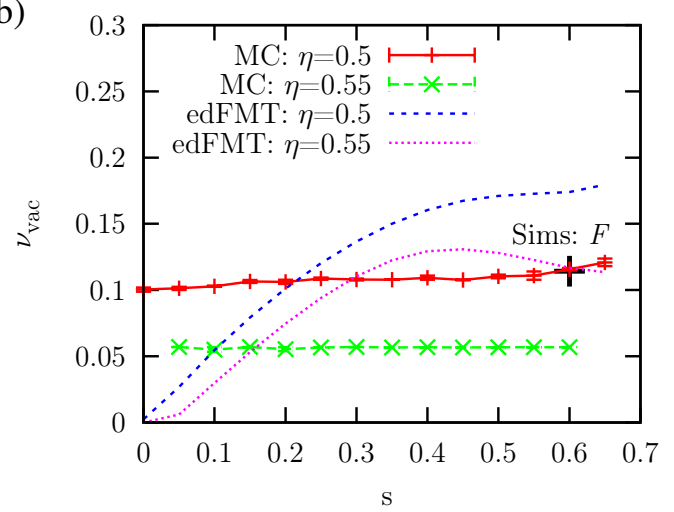

FIG. 5. (a) The vacancy concentration $\nu_{\mathrm{vac}}$ as a function of packing fraction $\eta$ as obtained from variable box edge length NVT simulations for parallel hard cubes, that is with $s=0$, from Cuesta et al's FMT ${ }^{[5}$ for parallel hard cubes and the edFMT of this work for rounded cubes with $s=10^{-4}$. (b) The same as (a), but now $s$ varies and $\eta$ is fixed to 0.5 or 0.55. The black points is determined by minimizing the free energy with respect to the vacancy concentration for $s=0$ and $\eta=0.47$ in (a) and for $s=0.6$ and $\eta=0.5$ in (b), see Fig. 2.

tion results are compared to FMT results obtained using the functional from this work and from Ref. 5. The agreement with the simulation data is reasonable for $s \simeq 0$, while the previous functiona ${ }^{5}$ for parallel hard cubes describes the simulation data the best. As $s$ increases, the agreement between theory and simulations deteriorates somewhat, and for large values of $s$ improves again, such that the difference in the compressibility factor resulting from the theory and the simulation is of the order of 1 for $s \gtrsim 0.1$.

The root mean squared deviation from the nearest lattice site (RMSD) as measured in event-driven molecular dynamics simulations (EDMD) is compared to FMT results in Figs. 7(a) and (b). Again, the first of these figures shows the $\eta$ dependence at $s=0$, while the second shows the $s$ dependence at fixed $\eta$. The RMSD is often compared with the Lindemann criterion ${ }^{55}$ for first order phase transitions, which says that the crystal starts to melts when the RMSD is around $10 \%-20 \%$ of the lat- (a)
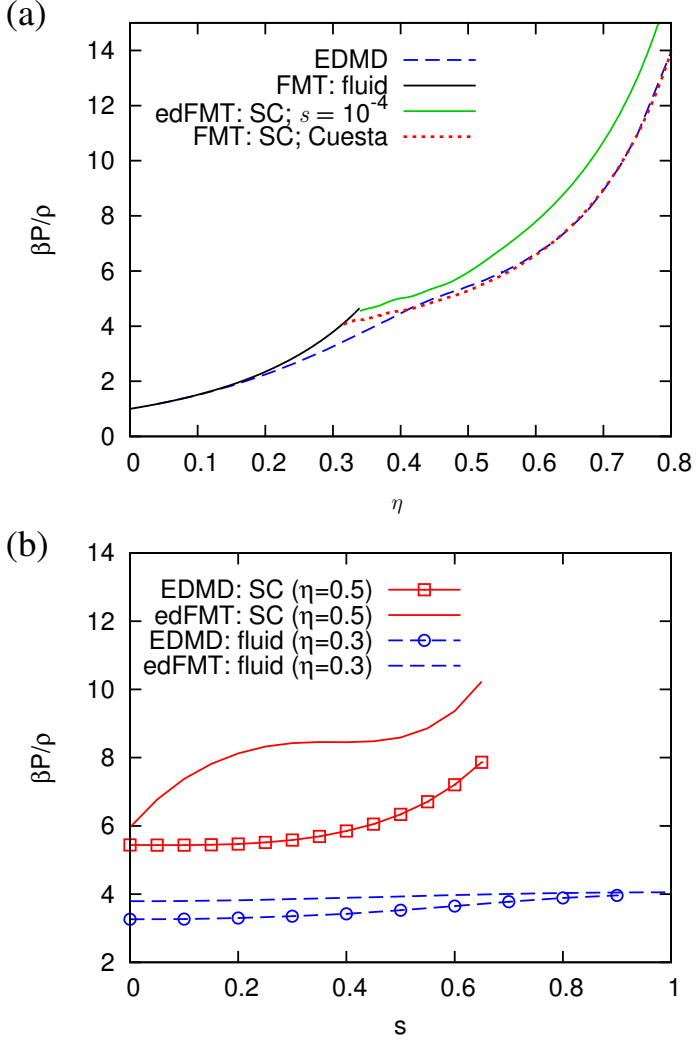

FIG. 6. (a) The compressibility factor $P / \rho k_{B} T$ as a function of packing fraction $\eta$ as obtained from event-driven MD simulations for parallel hard cubes $(s=0)$, from Cuesta et al's FMT $^{5}$ for parallel hard cubes and the edFMT of this work for rounded cubes with $s=10^{-4}$. The FMT and edFMT results for the fluid are exactly equal. (b) The same as (a), but now $s$ varies and $\eta$ is fixed to 0.3 , where the fluid is found, or 0.5 , where the simple cubic crystal is stable.

tice constant. As expected for second order phase transitions, the RMSD of the simple cubic crystal of (rounded) cubes is higher than the Lindemann parameter at the transition; it is in fact two to four times as high. The theoretical RMSD results at $s=0$ [Fig. 7(a)] again show the correct trend, but both theories under-estimate the simulation results, as was the case with the vacancy concentration. The result from cell theory (for zero vacancies) is also indicated by the think line. Interestingly, the simulation results have a substantially different slope than cell theory even when approaching close packing, the MSD from simulations is approximately 1.6 times the cell theory result. For comparison, the mean squared displacement measured in simulation of hard spheres is approximately $1.098(4 \sqrt{56}$ times the cell theory result. The dependence of the RMSD on the $s$ of FMT in Fig. 7(b) is qualitatively very similar to the simulation results: At $\eta=0.55$ the RMSD decreases monotonically, while the RMSD for $\eta=0.55$ shows a strong decrease with increasing $s$ for small $s$ followed by a small increase when $s$ is increased beyond a certain value $s \simeq 0.55$. 
(a)

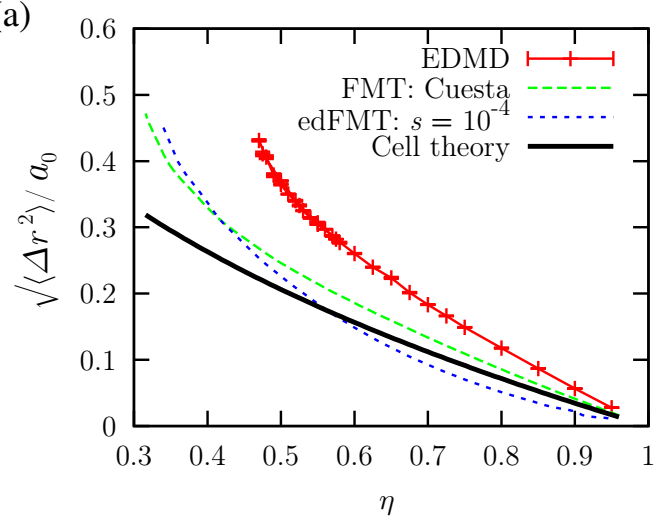

(b)

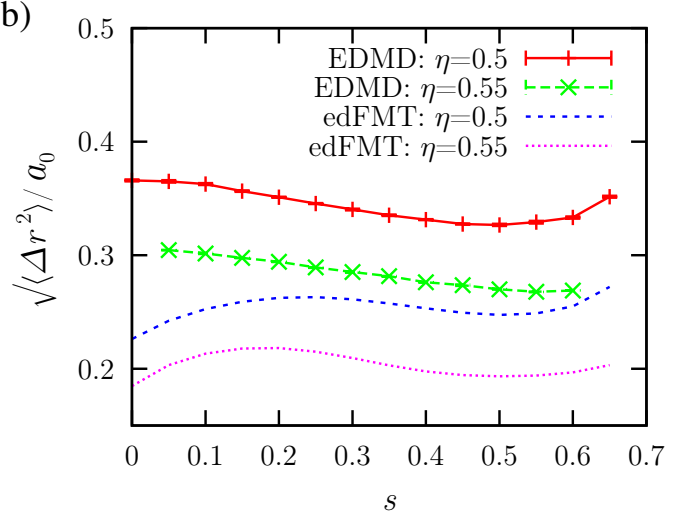

FIG. 7. The dimensionless root mean squared deviation (RMSD) from the nearest lattice site divided by the lattice constant, $\Delta r / a_{0}$, as obtained from variable box edge length NVT simulations. Results from our edFMT and the older FMT $^{7}$ are also shown. (a) The RMSD as a function of packing fraction $\eta$ for parallel hard cubes, that is, for $s=0$. The thick black line denotes the cell theory result without vacancies. (b) The RMSD for varying $s$ at $\eta=0.5$ and $\eta=0.55$.

\section{Phase diagram}

The phase diagram of parallel hard rounded cubes is shown in Fig. 8. The fluid to simple cubic (SC) crystal second order transitions for hard parallel cubes $s=0$ from this work have a critical packing fraction, $\eta_{c}=0.469(3)$ from simulations and $\eta_{c}=0.3325$ from FMT, which should be compared to the earlier simulation ${ }^{12}$ critical density, $\eta_{c}=0.53(1)$, and the result from the original $\mathrm{FMT}, \stackrel{8}{,} \eta=0.3143$. Our simulation results differ from the previous work because it was assumed that the SC crystal had zero vacancies in Ref. 12, In contrast, we find an extremely high vacancy concentration of $13 \%$ at coexistence. The FMT vacancy concentration of the earlier $\mathrm{FMT}^{[8}$ was $30 \%$, while our edFMT gives $23 \%$. Note, that the critical densities differ for the two theories and the simulations, which explains the reversal of the trends compared to the results at fixed packing fraction. Our FMT describes our simulation results slightly better than the earlier FMT,, 8 as far as the critical density and the vacancy concentration at $\eta_{c}$ are concerned. Conversely, the inclusion of vacancies has brought the critical density from simulations closer to the FMT results.

For hard parallel cubes with a finite rounding (i.e. $s \neq 0$ ), the transition from the fluid to the SC crystal phase is also second order both for the simulations and for FMT. For the simulations, this is indicated by the critical scaling whose exponents belong to the Heisenberg universality class $\frac{12}{12}$ The reasonable agreement between the simulation results and the FMT at $s=0$ is even slightly improved for $s \neq 0$, see Fig. 8 . The phases that were found using the simulations of single unit cells all have their separate area of stability in the phase diagram. At low $s$, the $\mathrm{SC}$ is stable at low densities, while the sheared variant (shSC) is stable at high densities. The transition from SC to shSC seems to become more weakly first order as $s$ approaches zero, and simultaneously the vacancy concentration decreases. For $s \leq 0.5$, we did not use free energy calculations, because the free energies of $\mathrm{SC}$ and $\mathrm{shSC}$ were very close making it hard to find the transition. Instead, we used direct simulations, which always lead to a pressure at which the difference in chemical potential was very small. As $s$ is increased, the $\mathrm{SC}-$ shSC transition goes down in density and at some point the shSC coexist directly with the fluid. Finally, the deformed FCC phase is stable for sphere-like particles $(s \geq 0.8)$. As mentioned in Sec. $\mathrm{VA}$ the def FCC phase actually consists of two crystals, one with a basecentered monoclinic (clino) unit cell and an other with a body-centered orthorhombic (ortho) unit cell. Our motivation for investigating the clino to ortho transition in this system, is that pyroxene, the second most abundant mineral in the earth's mantle, also has clino and ortho forms ${ }^{57}$ As a result, the clino-ortho transition of pyroxene is a topic of great interest in geology. In our case, the ortho-unit cell needs only be slightly deformed to form the clino unit cell: the angle between two of the orthorhombic lattice vectors is changed to slightly to a little more or less than 90 degrees; the difference is at maximum 2.22 degrees for $s=0.884$ at close packing. The packing fractions at the transition from clino to ortho and the transition from shSC to ortho were obtained by direct simulations, as shown in Fig. 8. The shSCortho phase transition is more strongly first order than the ortho-clino transition, which enabled us to calculate the free energy of the shSC and ortho phases separately and, reassuringly, the free energy difference between the two phases at the shSC-ortho transition, which was obtained in direct simulations, is smaller than the statistical error.

\section{CONCLUSIONS}

We studied a system of parallel rounded cubes (spherocubes) using fundamental measure theory and simulation with a special emphasis to the second order freezing into the simple cubic phase. We developed the fundamental measure theory starting from edFMT ${ }^{14115}$ expanding up to fourth order tensor terms and renormalizing the 

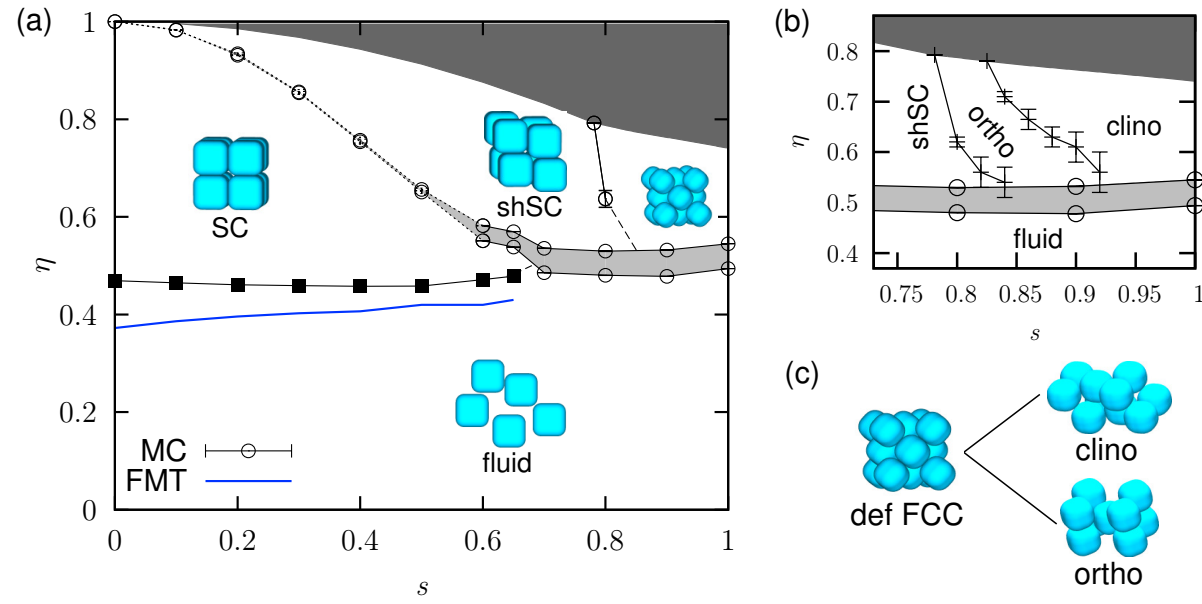

FIG. 8. (a) The phase diagram of parallel rounded cubes in the $\eta-s$ representation, where $\eta=v_{\text {rc }} N / V$ is the packing fraction with $v_{\mathrm{rc}}$ the volume of a rounded cube and $s=d / l$ is the rounding parameter (see Fig. 1). A cube has $s=0$ and a sphere $s=1$. Shown are the areas of stability of the deformed $f c c$ phase of near spheres (def FCC), the sheared cubic crystal (shSC), the simple cubic crystal (SC) and the fluid phase in white. The forbidden region above the close packing density is shown in dark gray and coexistence areas in lighter gray (coexistence lines are vertical). The filled symbols (MC simulations) and the thick line (FMT) denote second order phase transitions, while the empty symbols denote first order phase transitions from simulations. (b) An enlargement of the large $s$ region of the phase diagram: the def FCC phase is actually seen to have a body-centered orthorhombic variant (ortho) and a base-centered monoclinic variant (clino), as depicted in (c).

third and fourth order terms. When we apply the theory to the simple cubic phase, we find that the freezing is second order, not just for perfect cubes with shape parameter $s=0$, but also when we introduce a degree of rounding $s$ up to $s=0.65$. This finding is confirmed by finite size scaling techniques using Monte Carlo (MC) simulations. Furthermore, we find both in theory and simulations an unusually high vacancy concentration, namely $13 \%$, that is, about twice as high as for rotating perfect $\operatorname{cubes}^{13}$ and four orders of magnitude higher than that of hard spheres. $\frac{38 \mid 39}{}$ The very high vacancy concentration and the simple overlap criterion make this system an ideal system to study vacancies (the number of vacancies can always be decreased by increasing the density if so required).

When comparing the theory to the simulations, we find good qualitative agreement; exceptions are the dependence of the vacancy concentration and the pressure on the shape parameter $s$ which show the incorrect trend for FMT. Quantitative differences between the FMT and simulation results are found for most quantities. This is most likely caused by anomalous higher virial coefficients for this system (parallel cubes for instance have negative sixth and seventh virial coefficients 58 ) which are not reproduced by the theory. Nevertheless, the most important property, the packing fraction at freezing is predicted quite well by FMT over the whole range of $s$ where the simple cubic crystal is stable.

Finally, we completed the phase diagram of rounded cubes by investigating the possibility of other crystal phases in direct simulations and by performing free energy calculations using the results obtained in these sim- ulations. The phase diagram of parallel rounded cubes is surprisingly rich considering the simplicity of the model: it contains, apart from the simple cubic crystal phase, three more crystal phases. For low values of $s$ and high densities a sheared variant of the simple cubic (shSC) is found, while at low densities the simple cubic crystal is stable. For higher values of $s$, first the simple cubic crystal and later also the shSC phase disappears to be replaced by a body-centered orthorhombic crystal which is essentially a slightly deformed face-centered cubic crystal. Finally, a base-centered monoclinic crystal is found for values of $s$ near one, that is, for near spheres. The resulting symmetry change is interesting because a similar transition is found for an abundant mineral in the earth's mantle. ${ }^{[57}$ We expect that most, if not all, of the crystal phases we observed can also be found for rotating rounded cubes, which could be verified in experiments on colloidal rounded cubes.

\section{ACKNOWLEDGMENTS}

We thank Klaus Mecke, Marjolein Dijkstra, Laura Filion and Frank Smallenburg for useful discussions. This work was financially supported by the DFG within SFB TR6 (project D3). 


\section{Appendix A: Overlap algorithm and collision prediction}

The criterion for overlap between two (co-aligned) spherocubes is surprisingly simple: Two spherocubes overlap when shortest distance, $\Delta r_{i j}$, between any point on the surface of particle $i$ and any point on $j$ is smaller than $d$. The shortest distance can be calculated in the following two steps:

$$
\begin{aligned}
b_{i j, \nu} & \equiv\left|r_{j, \nu}-r_{i, \nu}\right|-\sigma \\
\Delta r_{i j, \nu} & =\left\{\begin{array}{cc}
\operatorname{sign}\left(r_{j, \nu}-r_{i, \nu}\right) b_{i j, \nu} & b_{i j, \nu} \geq 0 \\
0 & \text { otherwise }
\end{array}\right.
\end{aligned}
$$

the norm of the vector with components $\Delta r_{i j, \nu}$ for $\nu=$ $x, y, z$ is the shortest distance $\Delta r_{i j}$. The simplicity of the overlap criterion for spherocubes is a large advantage compared to superballs for which it can not be ascertained using analytical means whether two particles overlap or not. ${ }^{27}$ Note, that the overlap criterion for spherocubes becomes more complicated when the particles are not aligned with the Cartesian axes.

Collisions can be analytically predicted for parallel hard rounded cubes as follows: The surface of a rounded cube consists of sections of axis-aligned cylinders, planes and spheres, for which collisions can be easily calculated by the collision detection algorithms for the corresponding one, two and three dimensional hyper-spheres $[59$ Specifically, a collision test for two $n$-dimensional hyperspheres is required when exactly $n$ components of $\mathbf{b}_{i j}$ [the vector with components $b_{i j, \nu}$, see Eq.(A1)] are nonzero (at least one of components of $\mathbf{b}_{i j}$ is non-zero initially in an overlap-free configuration). The time at which a certain component of $\mathbf{b}_{i j}$ becomes nonzero can easily calculated from Eq. A1. All such times are determined, sorted and inserted in a list, whose subsequent elements define the time intervals at which certain parts of the surface of one of the particles might collide with a part of the other particle's surface. For each such a time interval, the corresponding hypersphere collision check is performed and the shortest of the resulting times is the time of collision of the two spherocubes. The rest of the algorithm is the same as the optimized algorithm for hard spheres, ${ }^{29}$ in which the collisions are stored, together with others event (such as measurements) in a binary tree leading to a theoretical $N \log (N)$ scaling of the computational effort for a fixed run time.29

\section{Appendix B: The Frenkel-Ladd method for crystals with vacancies}

In the original Frenkel-Ladd $\stackrel{41}{ }$ approach, each particle is coupled to its ideal lattice position with a harmonic spring, such that the external coupling potential reads:

$$
\beta U_{\text {har }}\left(\mathbf{r}^{N} ; \lambda\right)=\lambda \sum_{i=1}^{N}\left(\mathbf{r}_{i}-\mathbf{r}_{0, i}\right)^{2} / l^{2},
$$

where $\mathbf{r}_{i}$ denotes the position of particle $i$ and $\mathbf{r}_{0, i}$ the lattice site of particle $i$ and $\beta=1 / k_{B} T$. If the value of $\lambda$ is high enough or if the lattice positions are far enough apart, the particles do not interact and the free energy of the system is given by the known analytical free energy of the non-interacting Einstein crystal. $\stackrel{41}{\text { Therefore, the }}$ coupling constant $\lambda$ can be used to switch between an ideal Einstein crystal for high $\lambda$ and the unperturbed crystal for $\lambda=0$. The free energy of the crystal for $\lambda=0$ can then be found by integrating over $\lambda$ :

$$
f^{*}(N, V, T)=f_{\text {Einst }}^{*}(N, V, T)-\int_{0}^{\lambda_{\max }} \mathrm{d} \lambda\left\langle\frac{\partial f^{*}}{\partial \lambda}\right\rangle
$$

where $\left\langle\partial f^{*} / \partial \lambda\right\rangle=\left\langle U_{\text {har }}\left(\mathbf{r}^{N} ; \lambda\right)\right\rangle /(\lambda N)$. For $\lambda=0,\langle U\rangle$ diverges as the center of mass of the system diffuses as a whole, taking the particles ever further away from their lattice position. To overcome this problem, the center of mass is fixed which results in additional (small) terms in the free energy of the non-interacting system, which can be found in Refs. $28 \mid 41$. The value of $\lambda_{\max }$ required to obtain a non-interacting Einstein crystal depends on the lattice spacing, such that free energy calculations over a wide range of densities require constant tuning of $\lambda_{\max }$. However, the same value for $\lambda_{\max }$ can be used for every density if the inter-particle potential is replaced by a purely repulsive finite potential whose interaction strength is slowly decreased from essentially infinite to zero (where an essentially infinite interaction strength implies that no overlap is found during the simulation). The free energy difference between the interacting crystal for $\lambda=\lambda_{\max }$ and the non-interacting Einstein crystal is then obtained by integrating over the strength of interaction $\gamma$ of the inter-particle potential, see Refs. 6061 for details. The soft interaction between two particles, in this case, reads $\gamma\left(1-0.9 \Delta r_{i j} / l\right)$, see Eq. A2. We have used this method for all crystal phase with exception of the simple cubic crystal phase which had a large concentration of vacancies.

When the crystal has a nonzero vacancy concentration, the Frenkel-Ladd method $\stackrel{41}{~ r e q u i r e s ~ s o m e ~ m o d i f i c a t i o n s . ~}$ First of all, the particles are no longer associated with a single lattice site as they can hop to a different site when it is empty. Therefore, the harmonic potential can no longer be used. Instead, we use the periodic external potential proposed by Groh and Mulder, ${ }^{12}$ which reads

$$
\beta U_{\text {per }}\left(\mathbf{r}^{N}\right)=\lambda \sum_{i=1}^{N} \sum_{\nu=x, y, z} 1-\cos \left(k_{\nu} r_{i, \nu}\right),
$$

where $k_{\nu}=2 \pi N_{\nu} / L_{\nu}$. The number of unit cells $N_{\nu}$ in direction $\nu$ and the length $L_{\nu}$ of the edge of the box are adjusted to tune the density and vacancy concentration. Furthermore, we promote hopping of a particle 
from a filled lattice site to an empty one also at large $\lambda$ by performing moves of exactly one (cubic) lattice vector, which allows the distribution of vacancies over the lattice to equilibrate. Note, that the center of mass of the system is already fixed by the external potential, so no additional terms due to the fixing of the center of mass arise in the free energy. However, additional moves which translate the whole system homogeneously are required to efficiently equilibrate the center of mass. Finally, the ideal Einstein crystal free energy itself is modified because of the modified inter-particle potential. Additionally, the combinatorial free energy of choosing $N$ filled lattice sites out of a total of $M$ lattice sites needs to be included in the free energy. The total free energy of the non-interacting Einstein crystal reads

$$
f_{\text {Einst }}^{*}=-\frac{1}{N} \ln \left[\frac{M !}{(M-N) ! N !}\right]-3 \ln z_{1}\left(\lambda_{\max }\right)
$$

where $z_{1}(\lambda)=I_{0}(\lambda) \exp (-\lambda) a_{0} / \Lambda$ is the factor in the partition sum which results from the integration of the degrees of freedom of a single particle in one direction, and $I_{0}$ is the zeroth modified Bessel function of the first kind. If $\lambda$ goes to infinity, the external potential (B3) can approximated by a harmonic potential, and simultaneously $z_{1}(\lambda)$ approaches $\sqrt{\lambda / 2 \pi} a_{0} / \Lambda$ as $\lambda_{\max } \rightarrow \infty$. It is this expression for $z_{1}\left(\lambda_{\max }\right)$, which we use in practice, because the approximation has a negligible effect for the value of $\lambda_{\max }$ we used $\left(\lambda_{\max }=4000\right)$.

\section{Appendix C: Comparison with superballs}

To compare with superballs we need to relate Batten et al.'s aspect ratio $q^{27}$ to our $s$. The surface of a superball is described by the equation

$$
x^{2 q}+y^{2 q}+z^{2 q}=r^{2 q} .
$$

As mentioned in Ref. 62, from a family of shapes, such as super balls with varying $q$ and $r$, one shape can be selected that is most similar to the shape of interest, in this case, a spherocube with a certain value of $s$ (the size $l$ sets the length scale: only $r / l$ is relevant) by minimizing the so-called Hausdorff distance between the two shapes.

The Hausdorff distance is a distance on shape space that is commonly used in convex geometry ${ }^{63}$ In order to define the Hausdorff distance, we first define

$$
d^{\prime}(A, B)=\max _{\mathbf{x} \in A} \min _{\mathbf{y} \in B}|\mathbf{x}-\mathbf{y}|
$$

where $A$ and $B$ are solid (compact) bodies. The Hausdorff distance is then defined by

$$
d(A, B)=\max \left\{d^{\prime}(A, B), d^{\prime}(B, A)\right\} .
$$

In order to calculate the Hausdorff distance we need only consider those sets of points, such that each set has one point on the surface of $A$ and one on the surface of $B$ and

\begin{tabular}{c|cc} 
direction & $d_{\mathrm{sb}}$ & $d_{\mathrm{rc}}$ \\
\hline$(1,0,0)$ & $r$ & $l / 2$ \\
$(1,1,0) / \sqrt{2}$ & $r \sqrt{2}^{1-1 / q}$ & $\sigma / \sqrt{2}+d / 2$ \\
$(1,1,1) / \sqrt{3}$ & $r \sqrt{3}^{1-1 / q}$ & $\sqrt{3} \sigma / 2+d / 2$
\end{tabular}

TABLE I. The distance between the center of a particle and its surface in the indicated directions for superballs $\left(d_{s b}\right)$ and rounded cubes or spherocubes $\left(d_{\mathrm{rc}}\right)$.

the normals to the respective surfaces at these points are equal.

We only need to consider the distance from the center to the surface in this case in three different symmetry directions. These distances are listed in Tbl. I] To calculate the Hausdorff distance, we only need to maximize over the directions $\mathbf{n}$ given in Tbl. I

$$
d(\operatorname{sb}[r, q], \operatorname{rc}[l, s])=\max _{\mathbf{n}}\left|d_{\mathrm{sb}}(r, q)-d_{\mathrm{rc}}(l, s)\right|,
$$

where we use $\operatorname{sb}[r, q]$ and $\operatorname{rc}[l, s]$ to denote a superball and rounded cube, respectively, with a certain aspect ratio $(s$ or $q$ ) and linear size $(l$ or $r)$. Minimizing $d(\operatorname{sb}[r, q], \operatorname{rc}[l, s])$ for a certain value of $s$ with respect to $r / l$ and $q$, we obtain the superball that best fits a spherocube with aspect ratio $s$.

\section{REFERENCES}

${ }^{1}$ S. Brazovskii, Soviet Journal of Experimental and Theoretical Physics 41, 85 (1975).

${ }^{2}$ J. Fröhlich and C. Pfister, Commun. Math. Phys. 81, 277 (1981), 10.1007/BF01208901.

${ }^{3}$ For a review, see: K. J. Strandburg, Rev. Mod. Phys. 60, 161 (1988).

${ }^{4}$ T. R. Kirkpatrick, J. Chem. Phys. 85, 3515 (1986).

${ }^{5}$ J. A. Cuesta, Phys. Rev. Lett. 76, 3742 (1996).

${ }^{6}$ J. A. Cuesta and Y. Martínez-Ratón, J. Chem. Phys. 107, 6379 (1997).

${ }^{7}$ J. A. Cuesta and Y. Martínez-Ratón, Phys. Rev. Lett. 78, 3681 (1997).

${ }^{8}$ Y. Martínez-Ratón and J. A. Cuesta, J. Chem. Phys. 111, 317 (1999).

${ }^{9}$ Y. Rosenfeld, Phys. Rev. Lett. 63, 980 (1989).

${ }^{10}$ Y. Rosenfeld, M. Schmidt, H. Löwen, and P. Tarazona, Phys. Rev. E 55, 4245 (1997).

${ }^{11}$ E. A. Jagla, Phys. Rev. E 58, 4701 (1998).

${ }^{12}$ B. Groh and B. Mulder, J. Chem. Phys. 114, 3653 (2001).

${ }^{13}$ F. Smallenburg, L. Filion, M. Marechal, and M. Dijkstra, "Vacancy-stabilized crystalline order in hard cubes," To be published, arXiv:1111.3466 [condmat.soft].

${ }^{14}$ H. Hansen-Goos and K. Mecke, Phys. Rev. Lett. 102, 018302 (2009).

${ }^{15}$ H. Hansen-Goos and K. Mecke, J. Phys. Cond. Matt. 22, 364107 (2010). 
${ }^{16}$ A. Härtel and H. Löwen, J. Phys. Cond. Matt. 22, 104112 (2010).

${ }^{17}$ A. Härtel, R. Blaak, and H. Löwen, Phys. Rev. E 81, 051703 (2010).

${ }^{18}$ Y. Sun and Y. Xia, Science 298, 2176 (2002).

${ }^{19}$ V. N. Manoharan, M. T. Elsesser, and D. J. Pine, Science 301, 483 (2003).

${ }^{20}$ S. Glotzer and M. Solomon, Nat. Mater. 6, 557 (2007).

${ }^{21}$ S. Sacanna and D. J. Pine, Curr. Opin. Colloid. In. 16, 96 (2011).

${ }^{22}$ For a review and classification of the different shapes, see: R. Wittkowski and H. Löwen, Mol. Phys. (2012), published online: 10.1080/00268976.2011.609145.

${ }^{23}$ L. Rossi, S. Sacanna, W. T. M. Irvine, P. M. Chaikin, D. J. Pine, and A. P. Philipse, Soft Matter 7, 4139 (2011).

${ }^{24}$ S. Hernandez-Navarro, P. Tierno, J. Ignes-Mullol, and F. Sagues, Soft Matter 7, 5109 (2011).

${ }^{25}$ J. Y. Kim, F. E. Osterloh, H. Hiramatsu, R. K. Dumas, and K. Liu, J. Phys. Chem. B 109, 11151 (2005).

${ }^{26}$ H. Löwen, Physics Reports 237, 249 (1994).

${ }^{27}$ R. D. Batten, F. H. Stillinger, and S. Torquato, Phys. Rev. E 81, 061105 (2010).

${ }^{28}$ D. Frenkel and B. Smit, Understanding molecular simulation (Academic Press, 2002).

${ }^{29}$ D. C. Rapaport, Journal of Computational Physics 34, 184 (1980).

${ }^{30}$ B. M. Mulder, Mol. Phys. 103, 1411 (2005).

${ }^{31}$ L. Filion, M. Marechal, B. van Oorschot, D. Pelt, F. Smallenburg, and M. Dijkstra, Phys. Rev. Lett. 103, 188302 (2009).

${ }^{32}$ M. Parrinello and A. Rahman, Phys. Rev. Lett. 45, 1196 (1980).

${ }^{33}$ R. Najafabadi and S. Yip, Scripta Metall. 17, 1199 (1983).

${ }^{34}$ M. Marechal, R. J. Kortschot, A. F. Demirörs, A. Imhof, and M. Dijkstra, Nano Lett. 10, 1907 (2010).

${ }^{35}$ M. Marechal and M. Dijkstra, Phys. Rev. E 82, 031405 (2010).

${ }^{36}$ M. Marechal, A. Cuetos, B. Martínez-Haya, and M. Dijkstra, J. Chem. Phys. 134, 094501 (2011).

${ }^{37}$ J. de Graaf, R. van Roij, and M. Dijkstra, Phys. Rev. Lett. 107, 155501 (2011).

${ }^{38}$ C. H. Bennett and B. J. Alder, J. Chem. Phys. 54, 4796 (1971).

${ }^{39}$ M. Oettel, S. Görig, A. Härtel, H. Löwen, M. Radu, and T. Schilling, Phys. Rev. E 82, 051404 (2010).
${ }^{40}$ W. G. Hoover and F. H. Ree, J. Chem. Phys. 49, 3609 (1968).

${ }^{41}$ D. Frenkel and A. J. C. Ladd, J. Chem. Phys. 81, 3188 (1984).

${ }^{42}$ K. Binder, Rep. Prog. Phys. 60, 487 (1997).

${ }^{43}$ K. Chen, A. M. Ferrenberg, and D. P. Landau, Phys. Rev. B 48, 3249 (1993).

${ }^{44}$ R. Evans, Adv. Phys. 28, 143 (1979).

${ }^{45}$ R. Roth, R. Evans, A. Lang, and G. Kahl, J. Phys. Cond. Matt. 14, 12063 (2002).

${ }^{46}$ H. Hansen-Goos and R. Roth, J. Phys. Cond. Matt. 18, 8413 (2006).

${ }^{47}$ For a review, see R. Roth, J. Phys. Cond. Matt. 22, 063102 (2010); P. Tarazona, J. Cuesta, and Y. Martínez-Ratón, in Theory and Simulation of HardSphere Fluids and Related Systems, Lecture Notes in Physics, Vol. 753, edited by . Mulero (Springer Berlin / Heidelberg, 2008) pp. 247-341.

${ }^{48}$ Y. Rosenfeld, Mol. Phys. 86, 637 (1995).

${ }^{49}$ For an application of edFMT to dumbbells, see: M. Marechal, H. H. Goetzke, A. Härtel, and H. Löwen, J. Chem. Phys. 135, 234510 (2011).

${ }^{50}$ P. Tarazona, Phys. Rev. Lett. 84, 694 (2000).

${ }^{51}$ B. M. Mulder, Mol. Phys. 103, 1411 (20 May 2005).

${ }^{52}$ J. K. Percus and G. J. Yevick, Phys. Rev. 110, 1 (1958).

${ }^{53}$ R. W. Zwanzig, J. Chem. Phys. 24, 855 (1956).

${ }^{54}$ Y. Jiao, F. H. Stillinger, and S. Torquato, Phys. Rev. E 79, 041309 (2009).

${ }^{55}$ F. A. Lindemann, Phys. Z 11, 609 (1910).

${ }^{56}$ D. A. Young and B. J. Alder, J. Chem. Phys. 60, 1254 (1974).

${ }^{57} \mathrm{P}$. Ulmer and R. Stalder, American Mineralogist 86, 1267 (2001).

${ }^{58}$ W. G. Hoover and A. G. D. Rocco, J. Chem. Phys. 36, 3141 (1962).

${ }^{59}$ B. J. Alder and T. E. Wainwright, J. Chem. Phys. 31, 459 (1959).

${ }^{60}$ A. Fortini and M. Dijkstra, J. Phys. Cond. Matt. 18, L371 (2006).

${ }^{61}$ M. Marechal and M. Dijkstra, Phys. Rev. E 77, 061405 (2008).

${ }^{62}$ M. Marechal, A. Patti, M. Dennison, and M. Dijkstra, "Frustration of the isotropic-columnar phase transition of colloidal hard platelets by a transient cubatic phase," To be published, arXiv:1112.1209 [cond-mat.soft].

${ }^{63}$ M. Moszyńska, Selected topics in convex geometry (Birkhäuser, Boston, 2006). 